\title{
Ambulatory blood pressure is better associated with target organ damage than clinic blood pressure in patients with primary glomerular disease
}

\author{
Ruo-wei Wen ${ }^{1,2 \dagger}$, Xiao-qiu Chen ${ }^{1,2 \dagger}$, Ye Zhu ${ }^{1,2}$, Jian-ting Ke ${ }^{1,2}$, Yi Du $^{1,2}$, Cheng Wang ${ }^{1,2^{*}}$ and Tan-qi Lou ${ }^{3}$
}

\begin{abstract}
Background: Blood pressure is an important and modifiable cardiovascular risk factor. Ambulatory blood pressure monitoring (ABPM) provides valuable prognostic information in patients with chronic kidney disease (CKD), yet little is known about the association of various types of BP measurements with target organ damage (TOD) in patients with primary glomerular disease. The goal of this study was to investigate whether ambulatory blood pressure is better associated with TOD than clinic blood pressure in patients with primary glomerular disease.

Methods: 1178 patients with primary glomerular disease were recruited in this cross-sectional study. TOD were assessed by the following 4 parameters: left ventricular mass index (LVMI or LVH, left ventricular hypertrophy), estimated glomerular filtration rate (eGFR $\left.<60 \mathrm{ml} / \mathrm{min} / 1.73 \mathrm{~m}^{2}\right)$, albumin-to-creatinine ratio (ACR $\geq 30 \mathrm{mg} / \mathrm{g}$ ) and carotid intima-media thickness (CIMT) or plaque. Receiver operating characteristic (ROC) curve and multivariate logistic regression analyses were used to evaluate the relationship between ambulatory or clinic systolic blood pressure (SBP) indexes and TOD.
\end{abstract}

Results: Among 1178 patients (mean age, 39 years,54\% men), 116, 458, 1031 and 251 patients had LVH, eGFR < 60 $\mathrm{ml} / \mathrm{min} / 1.73 \mathrm{~m}^{2}, A C R \geq 30 \mathrm{mg} / \mathrm{g}$ and $\mathrm{CIMT} \geq 0.9 \mathrm{~mm}$ or plaque respectively. Area under ROC curves for TOD in ambulatory SBP, especially nighttime SBP, was greater than that in clinic SBP $(P<0.05)$. Multivariate logistic regression analyses showed that $24 \mathrm{~h} \mathrm{SBP}$, daytime SBP and nighttime SBP were significantly associated with LVH, eGFR $<60 \mathrm{ml} / \mathrm{min} / 1.73 \mathrm{~m}^{2}$ and $A C R \geq 30 \mathrm{mg} / \mathrm{g}$ after adjustment for clinic SBP, while the association of clinic SBP was attenuated after further adjustment for nighttime SBP.

Conclusions: Ambulatory blood pressure, especially nighttime blood pressure, is probably superior to clinic blood pressure and has a significant association with TOD in primary glomerular disease patients.

Keywords: Ambulatory blood pressure, Clinic blood pressure, Target organ damage, Primary glomerular disease

\footnotetext{
* Correspondence: wt770716@163.com

${ }^{\dagger}$ Ruo-wei Wen and Xiao-qiu Chen contributed equally to this work.

'Division of Nephrology, Department of Medicine, the Fifth affiliated hospital

Sun Yat-Sen University, ZhuHai 519000, Guangdong, China

${ }^{2}$ Guangdong Provincial Key Laboratory of Biomedical Imaging, the Fifth affiliated hospital Sun Yat-Sen University, Zhuhai 519000, Guangdong, China

Full list of author information is available at the end of the article
}

(c) The Author(s). 2020 Open Access This article is licensed under a Creative Commons Attribution 4.0 International License, which permits use, sharing, adaptation, distribution and reproduction in any medium or format, as long as you give appropriate credit to the original author(s) and the source, provide a link to the Creative Commons licence, and indicate if changes were made. The images or other third party material in this article are included in the article's Creative Commons licence, unless indicated otherwise in a credit line to the material. If material is not included in the article's Creative Commons licence and your intended use is not permitted by statutory regulation or exceeds the permitted use, you will need to obtain permission directly from the copyright holder. To view a copy of this licence, visit http://creativecommons.org/licenses/by/4.0/. The Creative Commons Public Domain Dedication waiver (http://creativecommons.org/publicdomain/zero/1.0/) applies to the data made available in this article, unless otherwise stated in a credit line to the data. 


\section{Background}

Chronic kidney disease (CKD) is a worldwide public health problem [1]. In these patients, hypertension is prevalent and considered the leading risk factor for death, which contributes to $45 \%$ of male deaths and $46 \%$ of female deaths [2-4]. Hypertension is also among the most important modifiable risk factors for end-stage renal disease (ESRD). Therefore, appropriate evaluation and management of hypertension to achieve blood pressure (BP) goals in CKD patients is necessary and valuable.

Ambulatory blood pressure monitoring (ABPM) could provide detailed information on BP over a $24 \mathrm{~h}$ period, and it is unanimously recommended by guidelines for BP management [5-7]. Previously, we have reported the high prevalence and prognostic value of nighttime hypertension in CKD patients compared with clinic blood pressure [8-10]. Recent evidence from large-scale cohort study also suggests that higher 24-h and nighttime blood pressure measurements were significantly associated with greater risks of death and cardiovascular disease, even after adjusting for other office-based or ambulatory blood pressure measurements [11]. All these data suggested ABPM was better than clinic blood pressure when assessing target organ damage (TOD) and prognosis in CKD patients.

However, CKD patients with different etiologies, like primary glomerular disease and diabetic kidney disease, were enrolled in prior studies at the same time. Primary glomerular disease and diabetic kidney disease were two main causes of CKD in many countries. In previous studies, the percentage of patients with diabetic kidney disease or diabetes mellitus at enrollment was $11-65 \%$ [12-15]. Compared with non-diabetic kidney disease, patients with diabetic kidney disease showed different BP characteristics and had a worse prognosis [16, 17]. The systolic blood pressure control was worse and nondipping rhythm was quite common [16]. Once in the period of massive albuminuria, the progression rate of diabetic kidney disease to ESRD is about 14 times that of other renal diseases [17], indicating patients with diabetic kidney disease would have more severe subclinical TOD, so it might be a big difference on the priority of ABPM between patients with and without diabetic kidney disease. It is very important to evaluate various types of BP measurements, especially ABPM, and assess the strength of their associations with TOD, focusing on patients with primary glomerular disease considering primary glomerular disease continues to be the very common in our country [18]. Accordingly, the objective of this study is to investigate whether ambulatory blood pressure is better associated with TOD than clinic blood pressure in patients with primary glomerular disease.

\section{Methods}

\section{Study population}

The study protocol was approved by the ethics committee of our hospital and adhered to the Declaration of Helsinki. Informed consent was obtained from each participant. Patients (14-75 years) with primary glomerular disease proved by renal biopsy or clinic findings after exclusion of secondary renal damage factors, were included. Patients were excluded from the study in case of: 1) diabetes mellitus; 2) acute changes in the eGFR $>30 \%$ in the previous 3 months; 3) maintenance dialysis or history of kidney transplantation; 4) cardiovascular disorders (unstable angina pectoris, heart failure, lifethreatening arrhythmia, atrial fibrillation, stroke and grade III-IV retinopathy); 5) pregnancy; 6) night work or shift-work employment; 6) intolerance to ABPM or invalid ABPM data; 7) inability to communicate and comply with all of the study requirements; Finally, a total of 1178 patients from our hospitals were enrolled in this study (Fig. 1).

\section{Ambulatory and clinic blood pressure monitoring}

Ambulatory blood pressure monitoring was performed with the automated measurements programmed at 15min intervals during the daytime and 30-min intervals at night as previously $[8,19]$. Appropriate cuff size was chosen based on the arm circumference and directly placed on the non-dominant arm. The monitor was programmed to measure every $15 \mathrm{~min}$ during the day (7:00 am to $10: 00 \mathrm{pm})$, and every $30 \mathrm{~min}$ during the night (10: $00 \mathrm{pm}$ to $7: 00 \mathrm{am}$ ). Monitoring was performed on a working day. Patients were instructed to maintain their usual but not strenuous level of activity, and to keep motionless at the time of measurement. ABPM data were invalid in cases of: 1) $>30 \%$ of measurements were lacking; 2) $>3 \mathrm{~h}$ data were missing, 3) sleep time at night was $<6$ or $>12 \mathrm{~h}$ during monitoring [20].

Clinic BP was measured at the physician's office with a standard mercury sphygmomanometer after a 5-min rest in a sitting position. For all patients, sphygmomanometric measurements were recorded by the same physician, who was not aware of the results of ABP recordings. Reported values of clinic BP were the mean of 2 or 3 measurements at 1-2 min intervals, recorded during the 2 days in which the ABPM device was installed and removed.

\section{Cardiac, renal and carotid assessment}

Cardiac structure and function were assessed by 2 investigators trained for this purpose before starting the study. Linear measurements of interventricular septal wall thickness (IVSd), end-diastolic left ventricular internal dimension (LVIDd), and posterior wall thickness (PWTd) were obtained from M-mode tracings, using 2- 


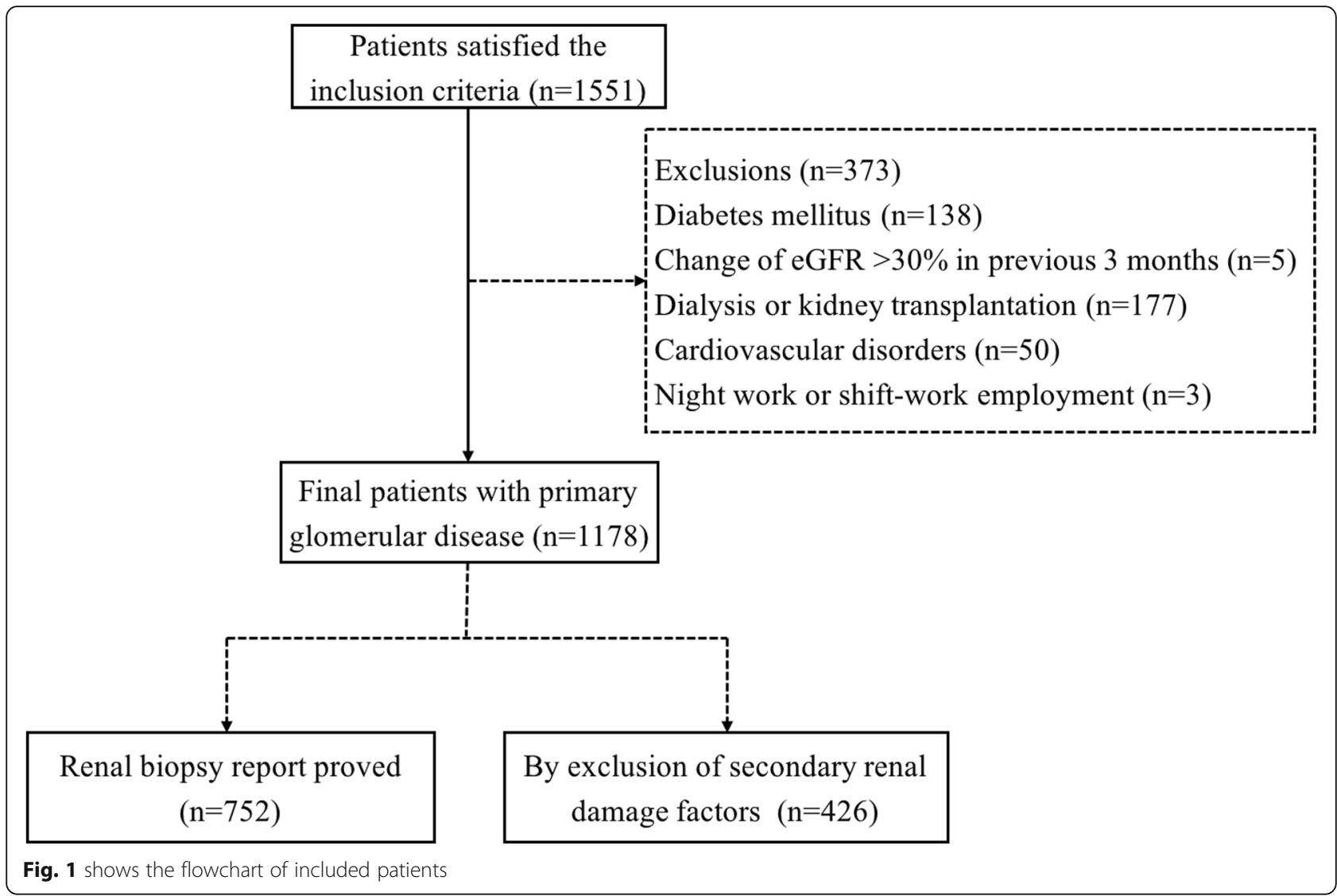

dimensional echocardiography. LVM was calculated using the Duvereux method [21]. The left ventricular mass index (LVMI) was obtained by calculating the ratio of LVM to body surface area.

Concentrations of serum creatinine (Scr) were measured by an enzymatic method traceable to isotope dilution mass spectrometry. The estimated Glomerular Filtration Rate (eGFR) was calculated using 2009 Chronic Kidney Disease Epidemiology Collaboration (CKD-EPI) creatinine equation [22]. Awaking (7:00 am to $10: 00 \mathrm{pm})$, bedtime (10:00 pm to $7: 00 \mathrm{am})$ and $24-\mathrm{h}$ urine samples were collected to predict excretion levels of urinary albumin, protein, and creatinine. Patients were asked to void their bladders at 7:00 am and 10:00 pm to ensure valid results.

Carotid intima-media thickness (cIMT) was determined by averaging 3 measurements taken on each carotid artery (in anterior, lateral and posterior directions), measuring the distance between the leading edge of the lumen-intima interface, and the leading edge of the collagenous upper layer of the adventitia using high-resolution B mode ultrasonography. Measurements were taken in areas free of obvious atherosclerotic plaques around the level of the carotid bifurcation.

\section{Collection of other data}

Information including age, sex, height, weight, smoking and alcohol consumption status, antihypertensive medication were obtained at the time of the BP measurement. Laboratory data (hemoglobin, albumin, calcium, phosphorus, intact parathyroid hormone, triglycerides, total cholesterol, high-density lipoprotein cholesterol, lowdensity lipoprotein cholesterol, uric acid and blood urea nitrogen) were obtained at the initial study visit. Blood samples were taken in the morning and analyzed using a 7180 Biochemistry Autoanalyzer (Hitachi, Tokyo, Japan) with reagents from Roche Diagnostics (Mannheim, Germany).

\section{Definitions}

CKD was divided into 5 stages and defined as the presence of kidney damage or decreased renal function (eGFR $<60 \mathrm{~mL} / \mathrm{min}$ per $1.73 \mathrm{~m} 2$ ) for $\geq 3$ months according to the Kidney Disease: Improving Global Outcomes (KDIGO) 2012 clinical practice guideline. Clinic hypertension was defined as clinic blood pressure (BP) $\geq 140$ / $90 \mathrm{mmHg}$ and ambulatory blood pressure (ABP) was defined as 24-h BP $\geq 130 / 80 \mathrm{mmHg}$. Masked hypertension was defined as a normal clinic BP $(\leq 140 / 90 \mathrm{mmHg})$ and an elevated ABP $(>130 / 80 \mathrm{mmHg})$. White coat 
hypertension was regarded as increased clinic BP $(>140 /$ $90 \mathrm{mmHg})$ and normal ABP $(\leq 130 / 80 \mathrm{mmHg})$. Normotension was defined as both clinic BP $<140 / 90 \mathrm{mmHg}$ and $\mathrm{ABP}<130 / 80 \mathrm{mmHg}$; Sustained hypertension was regarded as clinic $B P \geq 140 / 90 \mathrm{mmHg}$ and $A B P \geq 130 / 80$ $\mathrm{mmHg}$. Nighttime hypertension was defined as nighttime systolic BP (SBP) $\geq 120 \mathrm{mmHg}$ or/and diastolic BP (DBP) $\geq 70 \mathrm{mmHg}$. Isolated nighttime hypertension was defined as daytime $\mathrm{BP}<135 / 85 \mathrm{mmHg}$ and nighttime BP $\geq 120 / 70 \mathrm{mmHg}$. Participants with a reduction in SBP of $\geq 10 \%$ at night-time compared with daytime were considered to have a "dipper" pattern, and an "extreme dipper pattern" referred to a $>20 \%$ reduction at nighttime. A "non-dipper" pattern referred to $a<10 \%$ reduction at nighttime and a "reversed dipper pattern" referred to higher SBP at nighttime compared with daytime. Target organ damage (TOD) was defined if it met any of four conditions [23]: 1) left ventricular hypertrophy (LVH), namely LVMI $\geq 125 \mathrm{~g} / \mathrm{m} 2$ (man) or $\geq 120 \mathrm{~g} / \mathrm{m} 2$ (woman); 2) eGFR $<60 \mathrm{~mL} / \mathrm{min}$ per $1.73 \mathrm{~m}^{2}$; 3) Urinary albuminto-creatinine ratio (ACR) $\geq 30 \mathrm{mg} / \mathrm{g} ; 4$ ) cIMT $\geq 0.9 \mathrm{~mm}$ or existence of carotid plaque in ultrasonography.

\section{Statistical analysis}

Statistical analysis was performed with SPSS 25.0 (IBM Corp., Armonk, NY) and Medcalc 18.9(Broekstraat, Mariakerke, Belgium). Descriptive statistics were mean \pm SD for continuous variables or median (25-75th interquartile range) for non-normality variables. Frequency and percentage were used for categorical variables. To analyze the sensitivity and specificity of different BP indexes in relationship to TOD ( $\mathrm{LVH}$, eGFR $<60 \mathrm{ml} / \mathrm{min}$ per $1.73 \mathrm{~m}^{2}, \mathrm{ACR} \geq 30 \mathrm{mg} / \mathrm{g}$, cIMT $\geq 0.9 \mathrm{~mm}$ or carotid plaque), we generated and compared receiver operating characteristic (ROC) curves, including area under the curve (AUC) and their 95\% CIs. Considering each TOD may be affected by other important factors, and clinic and ambulatory SBP may have different prognostic value, we established 12 multivariate adjusted logistic regression models in all. All these models in sequence could be divided to four parts according to the TOD categories. Model 1-3, 4-6, 7-9 and 10-12 corresponded with $\mathrm{LVH}, \mathrm{eGFR}<60 \mathrm{ml} / \mathrm{min}$ per $1.73 \mathrm{~m}^{2}, \mathrm{ACR} \geq 30 \mathrm{mg} /$ g, cIMT $\geq 0.9 \mathrm{~mm}$ or carotid plaque, respectively. Model 1 included adjustment for age, sex, BMI, smoking, alcohol consumption status, hemoglobin. Albumin, eGFR, number of BP medications and type of glomerular disease. Model 4 included adjustment for age, sex, BMI, smoking, alcohol consumption status. Hemoglobin. albumin, ACR, iPTH, uric acid, calcium* phosphate product, number of BP medications and type of glomerular disease. Model 7 included adjustment for age, sex, BMI, smoking, alcohol consumption status, hemoglobin, albumin, uric acid, number of BP medications and type of glomerular disease. Model 10 included adjustment for age, sex, BMI, smoking, alcohol consumption status, eGFR, LDL-C, statin use, number of BP medications and type of glomerular disease. Model 2,5,8,11 included adjustment for the variables in Model 1,4,7,10 respectively and additional adjustment for clinic SBP when examining $24 \mathrm{~h} /$ daytime/nighttime SBP as the independent variable. Model 3,6,9,12 included adjustment for the variables in Model 1,4,7,10 respectively and additional adjustment for nighttime SBP when examining clinic $\mathrm{SBP}$ as the independent variable. Odds ratios of clinic SBP, 24-h SBP, daytime SBP and nighttime SBP, were shown per $1 \mathrm{SD}$ increase separately, so as to make them comparable. Probability values were 2 -tailed and $P<0.05$ was considered statistically significant for all comparisons.

\section{Results}

Demographic and clinical characteristics of the study population

Mean age of the study population was 38.8 years, and $53.7 \%$ was male. A total of 752 patients (63.8\%) had renal biopsy reports. The number of patients with $\operatorname{IgA}$ nephropathy, mesangial proliferative glomerulonephritis (MsPGN), minimal change disease (MCD), membranous nephropathy (MN); focal segmental glomerulosclerosis (FSGS) and membranoproliferative glomerulonephritis (MPGN) was 354, 17, 38, 162, 36 and 9, respectively; $18.5 \%$ of patients were current smokers, and101 patients (8.6\%) consumed alcohol. The prevalence of $\mathrm{LVH}$, eGFR $<60 \mathrm{ml} / \mathrm{min} / 1.73 \mathrm{~m}^{2}, \quad$ ACR $\geq 30 \mathrm{mg} / \mathrm{g}, \quad$ сIMT $\geq 0.9$ $\mathrm{mm}$ or plaque was $9.8,38.9,87.5,21.3 \%$, respectively (Table 1).

\section{Characteristics of ABPM in the study population}

The prevalence of nighttime hypertension in these patients was $62.2 \%$, while 272 patients $(23.1 \%)$ had isolated nighttime hypertension. A total of 609 (51.7\%) patients had non dipper pattern and 197 (16.7\%) patients had reversed dipper pattern, while only 341 (28.9\%) patients had a dipper pattern. 129(10.9\%) of patients had whitecoat hypertension and 175 (14.9\%) had masked hypertension. (Table 2) Mean BP in each category was shown in supplementary Table 1.

\section{Receiver-operating curve analysis of target organ damages}

In receiver-operating curve analysis, all SBPs were significantly associated with $\mathrm{LVH}$. Areas under the curve (AUC) were $0.779,0.770,0.760,0.721$ for nighttime SBP, $24 \mathrm{~h}$ SBP, daytime SBP and clinic SBP respectively. What's more, nighttime and 24h SBP ROC curves had greater AUC compared with clinic SBP in detecting the association with LVH $(\mathrm{P}<0.05)$. 
Table 1 Demographic characteristics and clinical parameters of study population

\begin{tabular}{|c|c|}
\hline Parameters & Value \\
\hline No. of Patients & 1178 \\
\hline Age (years) & $38.8 \pm 14.0$ \\
\hline Male $[n(\%)]$ & $633(53.7)$ \\
\hline BMI $\left(k g \cdot m^{-2}\right)$ & $22.9 \pm 3.5$ \\
\hline Smoker $[\mathrm{n}(\%)]$ & $218(18.5)$ \\
\hline Drinker $[n(\%)]$ & $101(8.6)$ \\
\hline \multicolumn{2}{|l|}{ Primary Glomerular Diseases } \\
\hline $\lg A[n(\%)]$ & $354(30.1)$ \\
\hline MsPGN [n(\%)] & $17(1.4)$ \\
\hline $\operatorname{MCD}[n(\%)]$ & $38(3.2)$ \\
\hline $\mathrm{MN}[\mathrm{n}(\%)]$ & $162(13.8)$ \\
\hline FSGS $[n(\%)]$ & $36(3.1)$ \\
\hline MPGN [n(\%)] & $9(0.8)$ \\
\hline Others [n(\%)] & $562(47.7)$ \\
\hline \multicolumn{2}{|l|}{ Medication } \\
\hline ACEI or ARB [n(\%)] & $576(48.9)$ \\
\hline$\beta$-blocker $[n(\%)]$ & $179(15.2)$ \\
\hline CCB $[n(\%)]$ & $362(30.6)$ \\
\hline a-blocker [n(\%)] & $71(6.0)$ \\
\hline Statin $[n(\%)]$ & $200(17.0)$ \\
\hline \multicolumn{2}{|l|}{ Antihypertensive medication use } \\
\hline $0[n(\%)]$ & $347(29.5)$ \\
\hline $1[n(\%)]$ & $570(48.4)$ \\
\hline $2[n(\%)]$ & $172(14.6)$ \\
\hline $3[n(\%)]$ & $78(6.6)$ \\
\hline $4[n(\%)]$ & $11(0.9)$ \\
\hline \multicolumn{2}{|l|}{ Laboratories } \\
\hline Hemoglobin $[\mathrm{g} / \mathrm{L}]$ & $124.8 \pm 27.6$ \\
\hline Albumin $[\mathrm{g} / \mathrm{L}]$ & $34.5 \pm 9.0$ \\
\hline Total cholesterol [mmol/L] & $5.3(4.3,6.9)$ \\
\hline LDL cholesterol $[\mathrm{mmol} / \mathrm{L}]$ & $3.2(2.4,4.4)$ \\
\hline HDL cholesterol $[\mathrm{mmol} / \mathrm{L}]$ & $1.2(1.0,1.5)$ \\
\hline Triglycerides $[\mathrm{mmol} / \mathrm{L}]$ & $1.5(1.0,2.3)$ \\
\hline Calcium [mg/dL] & $8.8 \pm 0.8$ \\
\hline Phosphate [mg/dL] & $3.7 \pm 0.4$ \\
\hline Calcium* Phosphate $\left[\mathrm{mg}^{2} / \mathrm{dL}^{2}\right]$ & $35.3 \pm 9.9$ \\
\hline iPTH [pmol/L] & $4.9(3.4,8.5)$ \\
\hline Uric acid $[\mu \mathrm{mol} / \mathrm{L}]$ & $434.0(346.3522 .9)$ \\
\hline Creatinine $[\mu \mathrm{mol} / \mathrm{L}]$ & $97.0(68.3200 .0)$ \\
\hline Stage $1[n(\%)]$ & $489(42.4)$ \\
\hline Stage $2[n(\%)]$ & $231(19.6)$ \\
\hline Stage $3[n(\%)]$ & $165(14.0)$ \\
\hline Stage $4[n(\%)]$ & $96(8.1)$ \\
\hline Stage $5[n(\%)]$ & $197(16.7)$ \\
\hline
\end{tabular}


Table 1 Demographic characteristics and clinical parameters of study population (Continued)

\begin{tabular}{lc}
\hline Parameters & Value \\
\hline eGFR-EPI $\left(\mathrm{ml} / \mathrm{min} / 1.73 \mathrm{~m}^{2}\right)$ & $78.0(30.0,108.0)$ \\
$\mathrm{eGFR}<60 \mathrm{ml} / \mathrm{min} / 1.73 \mathrm{~m}^{2}[\mathrm{n}(\%)]$ & $458(38.9)$ \\
$\mathrm{ACR}[\mathrm{mg} / \mathrm{g}]$ & $302.4(85.6851 .2)$ \\
$\mathrm{ACR} \geq 30 \mathrm{mg} / \mathrm{g}[\mathrm{n}(\%)]$ & $1031(87.5)$ \\
$\mathrm{CIMT}-$ left $[\mathrm{mm}]$ & $0.7 \pm 0.2$ \\
CIMT-right $[\mathrm{mm}]$ & $0.7 \pm 0.2$ \\
CIMT $\geq 0.9 \mathrm{~mm}$ or plaque $[\mathrm{n}(\%)]$ & $251(21.3)$ \\
Left ventricular mass index $\left[\mathrm{g} / \mathrm{m}^{2}\right]$ & $92.1 \pm 24.4$ \\
Left ventricular hypertrophy $[\mathrm{n}(\%)]$ & $116(9.8)$
\end{tabular}

Numbers are mean $\pm S D$, median (25-75th interquartile range) or number (percentage). MsPGN Mesangial. proliferative glomerulonephritis, $M C D$ Minimal change disease, MN Membranous nephropathy, FSGS Focal segmental glomerulosclerosis, MPGN Membranoproliferative glomerulonephritis, ACEI Angiotensin-converting enzyme inhibitor, $A R B$ Angiotensin receptor blocker, CCB Calcium channel blocker, LDL Low-density lipoprotein, HDL High-density lipoprotein, iPTH Intact parathyroid hormone, $A C R$ Albumin-to-creatinine ratio, CIMT Carotid. intima-media thickness

When detecting the association with eGFR $<60 \mathrm{ml} /$ $\mathrm{min} / 1.73 \mathrm{~m}^{2}$, AUC were $0.756,0.762,0.756,0.725$ for nighttime SBP, 24h SBP, daytime SBP and clinic SBP respectively, and statistical analysis showed daytime, nighttime and 24 h SBP had great AUC compared with clinic SBP in detecting the association with $\mathrm{eGFR}<60$ $\mathrm{ml} / \mathrm{min} / 1.73 \mathrm{~m}^{2}(\mathrm{P}<0.05)$.

Table 2 Clinic and ambulatory blood pressure characteristics in study population

\begin{tabular}{ll}
\hline Parameters & Value \\
\hline Clinic SBP $(\mathrm{mmHg})$ & $135.9 \pm 23.1$ \\
Clinic DBP $(\mathrm{mmHg})$ & $85.8 \pm 14.4$ \\
$24 \mathrm{~h} \mathrm{SBP}(\mathrm{mmHg})$ & $126.3 \pm 16.7$ \\
$24 \mathrm{~h} \mathrm{DBP}(\mathrm{mmHg})$ & $78.9 \pm 11.3$ \\
Daytime SBP $(\mathrm{mmHg})$ & $127.8 \pm 16.7$ \\
Daytime DBP $(\mathrm{mmHg})$ & $80.3 \pm 11.4$ \\
Nighttime SBP $(\mathrm{mmHg})$ & $119.6 \pm 18.4$ \\
Nighttime DBP $(\mathrm{mmHg})$ & $73.7 \pm 12.7$ \\
Nighttime hypertension [n(\%)] & $733(62.2)$ \\
Isolated nighttime hypertension [n(\%)] & $272(23.1)$ \\
Circadian patterns & \\
$\quad$ Reverse dipper [n(\%)] & $197(16.7)$ \\
$\quad$ Non dipper $[\mathrm{n}(\%)]$ & $609(51.7)$ \\
$\quad$ Dipper [n(\%)] & $341(28.9)$ \\
$\quad$ Extreme dipper [n(\%)] & $31(2.6)$ \\
Clinic-ambulatory BP status & \\
Normotension [n(\%)] & $446(37.9)$ \\
White-coat HBP [n(\%)] & $129(10.9)$ \\
Masked HBP [n(\%)] & $175(14.9)$ \\
Sustained HBP [n(\%)] & $428(36.3)$ \\
\hline
\end{tabular}

When considering ACR $\geq 30 \mathrm{mg} / \mathrm{g}$, AUC were 0.671, 0.654, 0.647, 0.629 for nighttime SBP, 24h SBP, daytime SBP and clinic SBP respectively, and only nighttime SBP had great AUC compared with clinic SBP in detecting the association with $A C R \geq 30 \mathrm{mg} / \mathrm{g}$ by statistical analy$\operatorname{sis}(\mathrm{P}<0.05)$.

Finally, when detecting the association with cIMT $\geq 0.9$ $\mathrm{mm}$ or plaque, AUC were $0.680,0.681,0.676,0.694$ for nighttime SBP, 24 h SBP, daytime SBP and clinic SBP respectively, and statistical analysis did not show any difference between ambulatory SBP and clinic SBP in detecting the association with $\mathrm{cIMT} \geq 0.9 \mathrm{~mm}$ or plaque. (Fig. 2 and Table 3).

\section{Factors associated with target-organ damage by multivariate logistic regression analyses}

Multivariate logistic regression analyses were carried out to clarify factors associated with target-organ damage. Higher clinic and ambulatory BPs were significantly associated with higher prevalence of LVH, eGFR $<60 \mathrm{ml} /$ $\mathrm{min} / 1.73 \mathrm{~m}^{2}$, and $\mathrm{ACR} \geq 30 \mathrm{mg} / \mathrm{g} \quad(\boldsymbol{P}<0.05) .24 \mathrm{~h}$ SBP, daytime SBP and nighttime SBP were still significantly associated with $\mathrm{LVH}, \quad$ eGFR $<60 \mathrm{ml} / \mathrm{min} / 1.73 \mathrm{~m}^{2}$ and ACR $\geq 30 \mathrm{mg} / \mathrm{g}(\boldsymbol{P}<0.05)$ after adjustment by clinic SBP. However, the association of clinic SBP with $\mathrm{LVH}$, $\mathrm{eGFR}<60 \mathrm{ml} / \mathrm{min} / 1.73 \mathrm{~m}^{2}$ and ACR $\geq 30 \mathrm{mg} / \mathrm{g}(\boldsymbol{P}<0.05)$ was attenuated after further adjustment for nighttime SBP $(\boldsymbol{P}=0.290, \boldsymbol{P}=0.160, \boldsymbol{P}=0.323$, respectively $)$. With respect to cIMT $\geq 0.9 \mathrm{~mm}$ or plaque, ambulatory SBP or clinic SBP was not significant in multivariate adjusted models with clinic and $24 \mathrm{~h} /$ daytime/nighttime SBP included. (Table 4).

\section{Discussion}

In this cross section study, we explore and compare associations of different BP indexes with TOD in CKD 


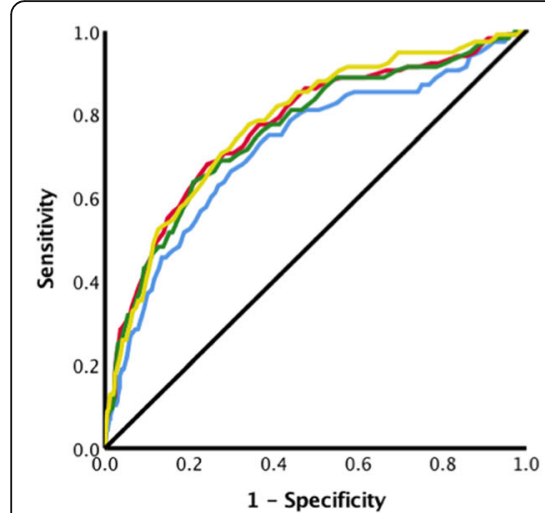

(A)LVH

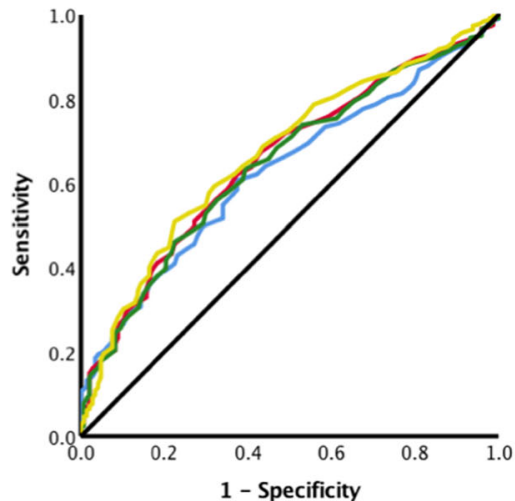

(C)ACR $\geq 30 \mathrm{mg} / \mathrm{g}$

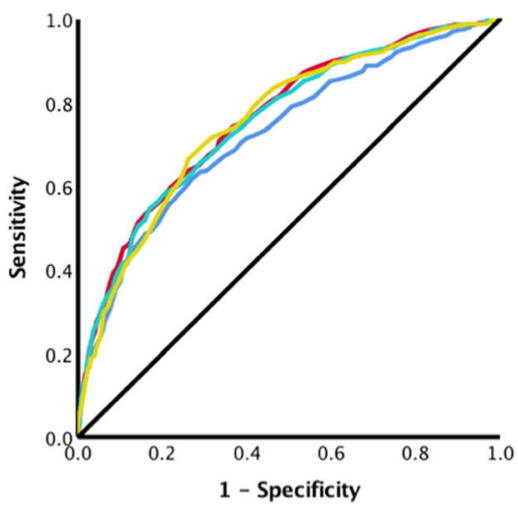

-Clinic SBP (0.725) — $24 \mathrm{~h} \mathrm{SBP}(0.762)$ - Daycime SBP (0.756) - Neference LiP 10.756 二Daytime SBP (0.760) -Nighttime SBP (0.779) 一 Reference Line

(B)eGFR $<60 \mathrm{ml} / \mathrm{min} / 1.73 \mathrm{~m}^{2}$

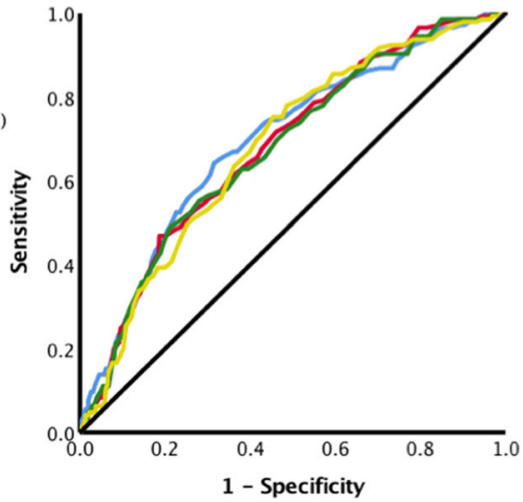

-Clinic SBP (0.694) - $24 \mathrm{~h} \mathrm{SBP}(0.681)$ - Daytime SBP (0.676) — Nighttime SBP (0.680) -24h SBP (0.654) - Daytime SBP (0.647) - Nighttime SBP (0.671)

(D)cIMT $\geq 0.9 \mathrm{~mm}$ or plaque

Fig. 2 shows receiver operating characteristic(ROC) curves of different BP indexes for TOD in four conditions: a left ventricular hypertrophy (LVH): LVMI $\geq 125 \mathrm{~g} / \mathrm{m} 2$ (man) or $\geq 120 \mathrm{~g} / \mathrm{m} 2$ (woman), b eGFR< $60 \mathrm{ml} / \mathrm{min}$ per $1.73 \mathrm{~m} 2$, c ACR $\geq 30 \mathrm{mg} / \mathrm{g}$, (D) clMT $\geq 0.9 \mathrm{~mm}$ or carotid plaque. Value in the bracket is the area under the curve of each line

patients with primary glomerular disease. We found that ambulatory SBP, especially nighttime SBP, was better associated with TOD than clinic SBP. What's more, higher $24 \mathrm{~h}$, daytime and nighttime SBP were significantly associated with TOD in these patients even adjusted clinic SBP in multivariate logistic regression analyses. All these data suggested that $A B P M$ is superior to clinic blood pressure in estimating TOD in patients with primary glomerular disease, and we should pay special attention to the use of ABPM in these patients in clinical practice.

Over the past years, ABPM developed into the recommended technique for BP measurement, risk stratification and classification of hypertension [24, 25]. Compared with clinic BP, ABPM increased the ability to

Table 3 Diagnostic performance of different BP indexes for TOD

\begin{tabular}{|c|c|c|c|c|}
\hline & \multicolumn{4}{|l|}{ TOD assessments } \\
\hline & LVH & eGFR $<60 \mathrm{ml} / / \mathrm{min} / 1.73 \mathrm{~m}^{2}$ & $A C R \geq 30 \mathrm{mg} / \mathrm{g}$ & cIMT $\geq 0.9 \mathrm{~mm}$ or plaque \\
\hline \multicolumn{5}{|l|}{ AUC $(95 \% \mathrm{Cl})$} \\
\hline Clinic SBP & $0.721(0.667,0.774)$ & $0.725(0.695,0.755)$ & $0.629(0.586,0.671)$ & $0.694(0.645,0.743)$ \\
\hline $24 \mathrm{~h} \mathrm{SBP}$ & $0.770(0.722,0.819)$ & $0.762(0.734,0.790)$ & $0.654(0.610,0.698)$ & $0.681(0.632,0.729)$ \\
\hline Daytime SBP & $0.760(0.711,0.810)$ & $0.756(0.728,0.784)$ & $0.647(0.603,0.691)$ & $0.676(0.627,0.725)$ \\
\hline Nighttime SBP & $0.779(0.733,0.824)$ & $0.756(0.728,0.784)$ & $0.671(0.627,0.715)$ & $0.680(0.632,0.728)$ \\
\hline \multicolumn{5}{|l|}{$P$ value, $Z$ value } \\
\hline $24 \mathrm{~h}$ vs. Clinic SBP & $0.048,1.977$ & $0.007,2.686$ & $0.204,1.272$ & $0.570,0.568$ \\
\hline Daytime vs. Clinic SBP & $0.115,1.578$ & $0.026,2.221$ & $0.368,0.900$ & $0.441,0.771$ \\
\hline Nighttime vs. Clinic SBP & $0.028,2.197$ & $0.037,2.083$ & $0.045,2.008$ & $0.587,0.543$ \\
\hline
\end{tabular}


Table 4 Univariate and multivariate logistic regression analysis of different BP indexes for TOD

\begin{tabular}{|c|c|c|c|c|}
\hline & \multicolumn{4}{|l|}{ Odds ratio $(95 \% \mathrm{Cl}), P$ value } \\
\hline & Clinic SBP (per 1 SD) & 24 h SBP (per 1 SD) & Daytime SBP (per 1 SD) & Nighttime SBP (per 1 SD) \\
\hline \multicolumn{5}{|l|}{ LVH } \\
\hline Unadjusted & $2.130(1.773,2.557),<0.001$ & $2.787(2.272,3.420),<0.001$ & $2.672(2.184,3.270),<0.001$ & $2.720(2.233,3.314),<0.001$ \\
\hline Model 1(M1) & $1.321(1.049,1.665), 0.018$ & $1.598(1.230,2.077),<0.001$ & $1.529(1.181,1.980), 0.001$ & $1.624(1.267,2.083),<0.001$ \\
\hline Model 2 (M1 + Clinic SBP) & - & $1.510(1.128,2.021), 0.006$ & $1.432(1.072,1.912), 0.015$ & $1.545(1.186,2.014), 0.001$ \\
\hline Model 3 (M1 + Nighttime SBP) & $1.145(0.891,1.472), 0.290$ & - & - & - \\
\hline \multicolumn{5}{|l|}{ eGFR $<60 \mathrm{ml} / / \mathrm{min}$ per $1.73 \mathrm{~m}^{2}$} \\
\hline Unadjusted & $2.537(2.189,2.942),<0.001$ & $3.003(2.573,3.504),<0.001$ & $2.899(2.490,3.376),<0.001$ & $2.863(2.455,3.337),<0.001$ \\
\hline Model 4(M4) & $1.265(0.991,1.615), 0.059$ & $1.457(1.145,1.854), 0.002$ & $1.415(1.115,1.796), 0.004$ & $1.492(1.181,1.885), 0.001$ \\
\hline Model 5 (M4 + Clinic SBP) & - & $1.416(1.073,1.867), 0.014$ & $1.365(1.036,1.798), 0.027$ & $1.446(1.123,1.863), 0.004$ \\
\hline Model 6 (M4 + Nighttime SBP) & $1.089(0.834,1.423), 0.529$ & - & - & - \\
\hline \multicolumn{5}{|l|}{$A C R \geq 30 \mathrm{mg} / \mathrm{g}$} \\
\hline Unadjusted & $1.684(1.373,2.065),<0.001$ & $1.810(1.474,2.221),<0.001$ & $1.753(1.433,2.145),<0.001$ & $1.981(1.597,2.457),<0.001$ \\
\hline Model 7(M7) & $1.409(1.080,1.837), 0.011$ & $1.546(1.194,2.002), 0.001$ & $1.501(1.164,1.935), 0.002$ & $1.730(1.326,2.256),<0.001$ \\
\hline Model 8(M7 + Clinic SBP) & - & $1.436(1.065,1.935), 0.018$ & $1.384(1.030,1.860), 0.031$ & $1.638(1.231,2.180), 0.001$ \\
\hline Model 9(M7 + Nighttime SBP) & $1.156(0.867,1.541), 0.323$ & - & - & - \\
\hline \multicolumn{5}{|l|}{$\mathrm{cIMT} \geq 0.9 \mathrm{~mm}$ or plaque } \\
\hline Unadjusted & $1.623(1.413,1.864),<0.001$ & $1.517(1.320,1.742),<0.001$ & $1.487(1.295,1.708),<0.001$ & $1.566(1.365,1.796),<0.001$ \\
\hline Model 10(M10) & $1.088(0.898,1.318), 0.389$ & $1.044(0.856,1.273), 0.669$ & $1.045(0.858,1.273), 0.663$ & $1.070(0.885,1.294), 0.482$ \\
\hline Model 11 (M10 + Clinic SBP) & - & $1.003(0.800,1.256), 0.981$ & $1.003(0.800,1.256), 0.981$ & $1.044(0.851,1.281), 0.681$ \\
\hline Model12(M10 + NighttimeSBP) & $1.071(0.870,1.317), 0.519$ & - & - & - \\
\hline
\end{tabular}

M1, M4, M7, M10 were short for Model 1, Model 4, Model 7 and Model 10, respectively. Model 1 included adjustment for age, sex, BMI, smoking, alcohol consumption status, hemoglobin. Albumin, eGFR, number of BP medications and type of glomerular disease. Model 4 included adjustment for age, sex, BMI, smoking, alcohol consumption status. Hemoglobin. albumin, ACR, iPTH, uric acid, calcium* phosphate product, number of BP medications and type of glomerular disease. Model 7 included adjustment for age, sex, BMI, smoking, alcohol consumption status, hemoglobin, albumin, uric acid, number of BP medications and type of glomerular disease. Model 10 included adjustment for age, sex, BMI, smoking, alcohol consumption status, eGFR, LDL-C, statin use, number of BP medications and type of glomerular disease. Model 2,5,8,11 included adjustment for the variables in Model 1,4,7,10 respectively and additional adjustment for clinic SBP when examining $24 \mathrm{~h} /$ daytime/nighttime SBP as the independent variable. Model 3,6,9,12 included adjustment for the variables in Model 1,4,7,10 respectively and additional adjustment for nighttime SBP when examining clinic SBP as the independent variable. Odds ratios in the table above present 1 SD increase in SBP

identify circadian variations in BP and identify daytime and nighttime BP. Prior studies have consistently demonstrated significant and superior association of ambulatory SBP with TOD in hypertensive patients $[26,27]$ as well as in CKD patients [13, 14]'. However, all these data were from CKD patients with different causes. CKD patients mixed with different etiologies like primary glomerular disease and diabetic kidney disease, were all included in these studies. Many factors such as glucose, inflammatory, salt intake would affect blood pressure status, so studies enrolled more diabetic patients would draw different conclusion compared with studies enrolled fewer diabetic patients. In previous studies, percentage of patients with diabetic kidney disease or diabetes mellitus at enrollment is up to 65\% [12-16]. As the high glucose influences the microenvironment of target organ, including heart, kidney and arteries, patients with diabetic kidney disease showed a more severe TOD, and progressed to ESRD more quickly than other renal disease, once in the period of massive albuminuria.
It reminds us of different meanings about ABPM in CKD patients with different etiologies. So we cannot directly extend these conclusions from patients with diabetic and non-diabetic kidney disease to patients with primary glomerular disease.

Primary glomerular disease is still predominant in hospitalized rural patients in China [28]. Data of ABPM in patients with primary glomerular disease was very limited and mostly compared with secondary or diabetic kidney disease in a small sample size [16, 29-32]. Previous study shows that patients with primary glomerular disease have a better control of BP and lower prevalence of abnormal circadian rhythm, when compared with diabetic kidney disease patients [16]. This reminds us probably there's a difference on the priority of ABPM between CKD patients with and without secondary glomerular disease, especially diabetic kidney disease. Moreover, associations of ABPM with TOD were poorly declared in past studies. Thus, after recruiting a large sample of these population, we evaluate various types of 
BP measurements, especially ABPM, and assess the strength of their associations with TOD.

The current study strengthened the notion that ABPM, especially nighttime BP carries valuable prognostic information in patients with primary kidney disease. These data confirmed the importance and superiority of ABPM in these patients. Future studies are required to ascertain whether individuals could benefit from BPlowering interventions targeting the ambulatory monitory results and ultimately reduce cardiovascular events.

Some limitations of our study deserve mention. Firstly, the size of the study population was not very large. Secondly, all enrolled CKD patients underwent only one ABPM and we could not rule out subsequent changes in ABPM. Thirdly, some patients with non-severe proteinuria or renal damage might have been excluded, leading to bias. Finally, we cannot infer a cause-effect relationship based on our cross-sectional data.

\section{Conclusion}

In conclusion, we have provided the first evidence that in CKD patients with primary glomerular disease, $24 \mathrm{~h}$, daytime, and nighttime SBP were better than clinic SBP and significantly associated with TOD, after adjustment for demographics and clinical characteristics. Thus, ABPM should be considered optimal and preferred measurement for estimating cardiovascular risk in these patients.

\section{Supplementary Information}

The online version contains supplementary material available at https://doi. org/10.1186/s12882-020-02200-1

Additional file 1: Supplementary Table 1. Mean blood pressure in each category of study population.

\section{Abbreviations}

ABPM: Ambulatory blood pressure monitoring; ACR: Albumin-to-creatinine ratio; ACEl: Angiotensin-converting enzyme inhibitor; ARB: Angiotensin receptor blocker; AUC: Area under the curve; BP: Blood pressure; CIMT: Carotid intima-media thickness; CCB: Calcium channel blocker; CKD: Chronic kidney disease; CKD-EPI: CKD Epidemiology Collaboration; DBP: Diastolic blood pressure; ESRD: End stage renal disease; eGFR: Estimated glomerular filtration rate; FSGS: Focal segmental glomerulosclerosis; HDL: High-density lipoprotein; HBP: High blood pressure; iPTH: Intact parathyroid hormone; LVMI: Left ventricular mass index; LVH: Left ventricular hypertrophy; LDL: Low-density lipoprotein; MsPGN: Mesangial proliferative glomerulonephritis; MCD: Minimal change disease; MN: Membranous nephropathy; MPGN: Membranoproliferative glomerulonephritis; ROC: Receiver operating characteristic; SBP: Systolic blood pressure; TOD: Target organ damage
}

\section{Acknowledgements}

We would like to thank all patients and their families for participating in this study.

\section{Authors' contributions}

Study concept and design: RWW, XQC CW. Acquisition, analysis, and interpretation of data: RWW, XQC, YZ, JT K, YD. Drafting of the manuscript: RWW, XQC. Critical revision of the manuscript for important intellectual content: RWW, XQC, TQL. Statistical analysis:.RWW, XQC. All authors read and approved the final manuscript.

\section{Funding}

This work was supported by Five-five Project of the Five Affiliated Hospital of Sun Yat-Sen University.

\section{Availability of data and materials}

The datasets used and analyzed during the current study are available from the corresponding author on reasonable request.

\section{Ethics approval and consent to participate}

Ethical approval was granted by the Ethics Committee of our hospital. Participants were required to provide written consent to participate. Written informed consent for participation in the study was obtained where participants are children (under 16 years old) from their parent or guardian.

\section{Consent for publication}

Not applicable.

\section{Competing interests}

The authors declare no conflict of interests. The results presented in this paper have not been published previously in whole or part, except in abstract format.

\section{Author details}

${ }^{1}$ Division of Nephrology, Department of Medicine, the Fifth affiliated hospital Sun Yat-Sen University, ZhuHai 519000, Guangdong, China. ${ }^{2}$ Guangdong Provincial Key Laboratory of Biomedical Imaging, the Fifth affiliated hospital Sun Yat-Sen University, Zhuhai 519000, Guangdong, China. ${ }^{3}$ Division of Nephrology, Department of Medicine, the Third affiliated hospital Sun Yat-Sen University, GuangZhou 510000, Guangdong, China.

Received: 12 December 2019 Accepted: 2 December 2020

Published online: 11 December 2020

References

1. Stenvinkel P. Chronic kidney disease: a public health priority and harbinger of premature cardiovascular disease. J Intern Med. 2010;268:456-67.

2. The Global Burden of Metabolic Risk Factors for Chronic Diseases Collaboration Writing and global analysis group. Cardiovascular disease, chronic kidney disease, and diabetes mortality burden of cardiometabolic risk factors from 1980 to 2010: a comparative risk assessment. Lancet Diabetes Endocrinol. 2014;2:634-47.

3. Benjamin EJ, Virani SS, Callaway CW, Chamberlain AM, Chang AR, Cheng S, et al. Heart disease and stroke statistics-2018 update: a report from the American Heart Association. Circulation. 2018;137:e467-92.

4. Sarafidis PA, Li S, Chen SC, et al. Hypertension awareness, treatment, and control in chronic kidney disease. Am J Med. 2008;121:332-40.

5. Whelton PK, Carey RM, Aronow WS, et al. 2017 ACC/AHA/AAPA/ABC/ACPM/ AGS/APhA/ASH/ASPC/ NMA/PCNA guideline for prevention, detection, evaluation, and management of high blood pressure in adults: a report of the American College of Cardiology/American Heart Association Task Force on Clinical Practice Guidelines. J Am Coll Cardiol. 2018;71(19):e127-248.

6. Williams B, Mancia G, Spiering W, ESC Scientific Document Group, et al. 2018 ESC/ESH guidelines for the management of arterial hypertension. Eur Heart J. 2018:39(33):3021-104.

7. Shimamoto K, Ando K, Fujita T, et al. Japanese Society of Hypertension Committee for Guidelines for the Management of Hypertension. The Japanese Society of Hypertension guidelines for the management of hypertension (JSH 2014). Hypertens Res. 2014;37(4):253-390.

8. Wang C, Deng WJ, Gong WY, et al. High prevalence of isolated nocturnal hypertension in Chinese patients with chronic kidney disease. J Am Heart Assoc. 2015;4(6):e002025

9. Wang C, Li Y, Zhang J, et al. Prognostic effect of isolated nocturnal hypertension in Chinese patients with nondialysis chronic kidney disease. J Am Heart Assoc. 2016;5(10):e004198.

10. Li Y, Deng Q, Li H, et al. Prognostic value of nighttime blood pressure load in Chinese patients with nondialysis chronic kidney disease. J Clin Hypertens (Greenwich). 2017;19(9):890-8. 
11. Yang WY, Melgarejo JD, Thijs L, et al. Association of Office and Ambulatory Blood Pressure with Mortality and cardiovascular outcomes. JAMA. 2019; 322(5):409-20.

12. Cha RH, Kim S, Ae Yoon S, et al. Association between blood pressure and target organ damage in patients with chronic kidney disease and hypertension: results of the APrODiTe study. Hypertens Res. 2014;37(2):172-

13. Drawz PE, Alper AB, Anderson AH, et al. Masked Hypertension and Elevated Nighttime Blood Pressure in CKD: Prevalence and Association with Target Organ Damage. Clin J Am Soc Nephrol. 2016;11(4):642-52 CJN.08530815.

14. Scheppach JB, Raff U, Toncar S, et al. Blood pressure pattern and target organ damage in patients with chronic kidney disease. Hypertension. 2018; 72(4):929-36.

15. Agarwal R. Arterial stiffness and its relationship to clinic and ambulatory blood pressure: a longitudinal study in non-dialysis chronic kidney disease. Nephrol Dial Transplant. 2017;32(11):1850.

16. Li J, Wang F, Jian G, et al. Analysis of 24-hour ambulatory blood pressure monitoring in patients with diabetic nephropathy: a hospital-based study. Clin Nephrol. 2013;79(3):199-205.

17. Hu RM, et al. Chinese expert consensus on prevention and treatment of diabetic nephropathy (2014) [in Chinese]. Chin J Diabetes Mellitus. 2014; 6(11):792-801.

18. Jha V, Garcia-Garcia G, Iseki K, et al. Chronic kidney disease: global dimension and perspectives. Lancet. 2013;382(9888):260-72.

19. Li X, Lian R, Zhu Y, et al. Masked morning hypertension correlated with target organ damage in non-dialysis patients with chronic kidney disease. J Hypertens. 2020;38(9):1794-801.

20. Parati G, Stergiou G, O'Brien E, et al. European Society of Hypertension practice guidelines for ambulatory blood pressure monitoring. J Hypertens. 2014;32:1359-66.

21. Devereux RB, Alonso DR, Lutas EM, Gottlieb GJ, Campo E, Sachs I, Reichek N. Echocardiographic assessment of left ventricular hypertrophy: comparison to necropsy findings. Am J Cardiol. 1986;57:450-8.

22. Levey AS, Stevens LA, Schmid CH, et al. A new equation to estimate glomerular filtration rate. Ann Intern Med. 2009:150:604-12

23. Liu LS. Writing group of 2010 Chinese guidelines for the management of hypertension. 2010 Chinese guidelines for the management of hypertension [in Chinese]. Chin J Hypertens. 2011;19(8):701-42.

24. Fan $H Q$, Li Y, Thijs $L$, et al. Prognostic value of isolated nocturnal hypertension on ambulatory measurement in 8711 individuals from 10 populations. J Hypertens. 2010;28:2036-45.

25. O'Brien E, Parati G, Stergiou G, et al. European Society of Hypertension Working Group on Blood Pressure Monitoring. European Society of Hypertension position paper on ambulatory blood pressure monitoring. J Hypertens. 2013;31(9):1731-68.

26. Gaborieau V, Delarche N, Gosse P. Ambulatory blood pressure monitoring versus self-measurement of blood pressure at home: correlation with target organ damage. J Hypertens. 2008;26:1919-27.

27. Bliziotis IA, Destounis A, Stergiou GS. Home versus ambulatory and office blood pressure in predicting target organ damage in hypertension: a systematic review and meta-analysis. J Hypertens. 2012;30:1289-99.

28. Zhang L, Long J, Jiang W, et al. Trends in chronic kidney disease in China. N Engl J Med. 2016;375(9):905-6.

29. Chen W. Clinical meaning of ambulatory blood pressure monitoring in patients with diabetic nephropathy [in Chinese]. Chin J Mod Drug Appl. 2012;14:34-5.

30. Zhang W, Yuan J, Yuan $\mathrm{H}$, et al. Comparison analysis of $24 \mathrm{~h}$ ambulatory blood pressure monitoring between primary and secondary glomerular disease [in Chinese]. J Shanghai Jiaotong Univ (medical science). 2013;33(4): 463-7.

31. Ying B, Shao X, Li Y, et al. Analysis of $24 \mathrm{~h}$ ambulatory blood pressure monitoring between primary and secondary glomerular disease in children [in Chinese]. Guizhou Med J. 2015;2:142-4.

32. Tamura K, Yamauchi J, Tsurumi-Ikeya Y, et al. Ambulatory blood pressure and heart rate in hypertensives with renal failure: comparison between diabetic nephropathy and non-diabetic Glomerulopathy. Clin Exp Hypertens. 2008;30(1):33-43.

\section{Publisher's Note}

Springer Nature remains neutral with regard to jurisdictional claims in published maps and institutional affiliations.

Ready to submit your research? Choose BMC and benefit from:

- fast, convenient online submission

- thorough peer review by experienced researchers in your field

- rapid publication on acceptance

- support for research data, including large and complex data types

- gold Open Access which fosters wider collaboration and increased citations

- maximum visibility for your research: over $100 \mathrm{M}$ website views per year

At $\mathrm{BMC}$, research is always in progress.

Learn more biomedcentral.com/submissions 